\title{
Association between early recurrences of atrial tachyarrhythmias and long-term outcomes in patients after repeat atrial fibrillation ablation
}

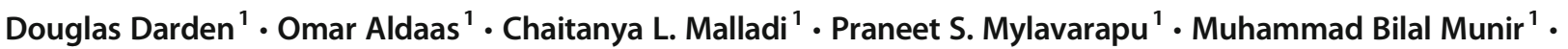 \\ Frederick T. Han ${ }^{1} \cdot$ Kurt S. Hoffmayer $^{1} \cdot$ Farshad Raissi $^{1} \cdot$ Gordon Ho $^{1} \cdot$ David Krummen $^{1} \cdot$ Gregory K. Feld $^{1}$. \\ Jonathan C. Hsu ${ }^{1}$ (D)
}

Received: 13 December 2020 / Accepted: 26 March 2021 / Published online: 7 April 2021

(C) The Author(s) 2021

\begin{abstract}
Purpose Early recurrence of atrial tachyarrhythmia (ER) is predictive of late recurrence of atrial tachyarrhythmia (LR) after firsttime atrial fibrillation (AF) ablation, but the association in patients undergoing repeat AF ablation is unknown. We aim to determine the incidence and prognostic significance of ER after repeat ablation.

Methods A total of 259 consecutive patients (mean age 64 years, 75.3\% male) undergoing repeat AF ablation with complete follow-up data were included at a single institution from 2010 to 2015. ER and LR were defined as atrial tachyarrhythmia (AF, atrial flutter or atrial tachycardia) > $30 \mathrm{~s}$ within the 3-month blanking period (BP) and after the 3-month BP, respectively.

Results ER occurred in 79/259 (30.5\%), and LR occurred in 138/259 (53\%) at a median follow-up of 1221 (IQR: 523-1712) days. Four-year freedom from LR was $22 \%$ and $56 \%$ in patients with and without ER, respectively $(p<0.001)$. After multivariate adjustment, ER was strongly associated with LR, cardioversion post BP, and repeat ablation, but not associated with hospitalization. Compared to those with no ER, there was a higher risk of LR when ER occurred within the first month of the BP [month 1: hazard ratio (HR) 2.32, confidence interval (CI) 1.57-3.74, $p<0.001$; month 2: HR 2.01, CI 1.13-3.83, $p=0.02$; month 3: HR 1.46, CI 0.5-3.36, $p=0.37$ ], however the prediction of LR based on timing within the BP was poor (area under curve 0.64).

Conclusion Following repeat AF ablation, ER is strongly associated with LR, cardioversion post BP, and repeat ablation.
\end{abstract}

Keywords Early recurrence $\cdot$ Blanking period $\cdot$ Repeat ablation $\cdot$ Pulmonary vein isolation $\cdot$ Atrial fibrillation

\section{Introduction}

Catheter ablation for drug-refractory symptomatic atrial fibrillation (AF) is an effective treatment; however, early recurrence of atrial tachyarrhythmias (ER) after successful pulmonary vein isolation (PVI) is common [1]. Although ER may occur in over a third of patients, it does not necessarily imply ablation failure [2]. Since not all patients with ER experience late recurrence of atrial tachyarrhythmias (LR), it is thought that ER may result from transient effects of post-ablation inflammation and autonomic imbalance [3]. Therefore,

Jonathan C. Hsu

jonathan.hsu@ucsd.edu

1 Division of Cardiology, Department of Medicine, University of California, San Diego, 9452 Medical Center Drive, 3rd Floor, Room 3E-417, La Jolla, CA 92037, USA consensus guidelines have proposed a 3-month post procedural blanking period (BP) in which recurrences should not prompt reintervention [1].

However, several studies involving patients following de novo PVI have reported a strong association between ER and LR [4-6]. Furthermore, the timing of ER within the BP has important predictive implications. Specifically, those with ER that occurs later in the $\mathrm{BP}$ are at a significantly higher risk of LR, advocating consideration of shortening of the BP duration [7-9].

Those with LR frequently pursue repeat ablation and clinicians subsequently repeatedly apply the recommended BP to this cohort. Although repeat ablation increases arrhythmiafree survival considerably, no previous study has examined the significance of ER in patients undergoing repeat ablation [10]. The aim of the present study is to determine the incidence, clinical predictors, and prognostic significance of ER and the timing of ER in patients undergoing repeat AF ablation. 


\section{Methods}

\subsection{Study population}

Consecutive patients undergoing repeat radiofrequency ablation for repeat atrial fibrillation with complete follow-up data at the University of California, San Diego (UCSD), Health System between October 2009 and March 2015 were analyzed from the UCSD AF Ablation Registry, an observational, retrospective cohort study. The UCSD AF Ablation Registry was designed as a clinical registry of all patients undergoing left atrial ablation procedures for atrial arrhythmias at UCSD, a single academic center, as captured by a procedural database (Perminova, Inc., San Diego, CA) to collect patient, provider, and intra-procedural characteristics. All AF ablation procedures captured by the registry were linked to clinical encounters as recorded by the electronic medical record at the institution (Epic, Verona, WI). Data on baseline demographics, medical history, laboratory data, medications, and cardiovascular implantable devices were collected. Intra-procedural registry reports were reviewed to determine fluoroscopy and procedure times and ablation lesion sets.

All patients undergoing repeat atrial fibrillation ablation had a history of symptomatic, medically refractory, paroxysmal or persistent AF after a first PVI ablation procedure. Patients with atypical atrial flutter and atrial tachycardia as the primary indication and those with $>2$ previous $\mathrm{AF}$ catheter ablations were excluded. The study was approved by the UCSD Institutional Review Board.

\subsection{Procedural details}

Informed consent was obtained prior to all ablation procedures. General anesthesia was used in all cases. Intravenous heparin was used to target an activated clotting time of 350$400 \mathrm{~s}$. A transseptal puncture was performed under direct visualization with intracardiac echocardiography guidance. Electroanatomic mapping systems were used in all cases (CARTO $^{\text {TM }}$ (Biosense-Webster Inc, Diamond Bar, CA; or Ensite $^{\mathrm{TM}}$, St Jude Medical, Inc., Minneapolis, MN). Esophageal position and temperature were monitored during all left atrial ablations using a multipolar temperature probe (Circa S-Cath, Circa Scientific, Inc., Englewood, CO) positioned in the esophagus behind the left atrium at the level of the ablation catheter, in order to avoid any temperature rise above $38{ }^{\circ} \mathrm{C}$. If recovery of conduction from PVs was observed, repeat PVI was performed using a segmental, circumferential, or both approaches. Additional lesion sets outside the previously ablated regions were performed at the discretion of the operator, including left atrial roof line, mitral valve isthmus line, coronary sinus ablation, and complex fractional atrial electrogram ablation. Additionally, organized atrial arrhythmias such as atrial flutter (AFL) or atrial tachycardia
(AT) were mapped and ablated, as were ectopic premature atrial contractions. Closed and open irrigated and noncontact and contact force sensing catheters were also used at the discretion of the operator. The endpoint of PVI was elimination of all $\mathrm{PV}$ potentials and demonstration entrance and exit block by pacing after a 30-min waiting period and elimination of a trigger or a line of bidirectional conduction block if adjunctive ablations were performed.

\subsection{Post-ablation follow-up}

After ablation, patients were hospitalized overnight and usually discharged the day after the procedure if stable. Oral anticoagulation was restarted in all patients after ablation. Most patients were discharged on the antiarrhythmic drug regimen they had been on before ablation and were most commonly maintained on this medication for up to 90 days, after which it was discontinued per provider discretion.

After discharge, all patients were seen in the UCSD outpatient arrhythmia clinic typically at 1, 6, 12, and 24 months after ablation and at 12-month intervals thereafter. A clinical assessment and a 12-lead electrocardiogram were routinely performed at each visit. Patients had routine ambulatory ECG monitoring (24-h Holter monitor, extended ambulatory electrocardiogram monitoring, or event monitoring) at 6,12 , and 24 months after ablation. Additional monitoring was provided in any patient reporting symptoms suggesting recurrent arrhythmia.

\subsection{Outcomes}

Documentation of any arrhythmic episode was based on ECG, Holter or event monitor data, or implantable device recordings. ER was defined as any atrial tachyarrhythmia (AF, AFL, AT) lasting $>30 \mathrm{~s}$ within the first 3 months after the repeat ablation procedure. LR was defined as recurrence of any atrial tachyarrhythmia (AF, AFL, AT) $>30$ s occurring $>3$ months after repeat procedure.

Additional clinical outcomes were determined during follow-up and included in-hospital adverse events, direct current cardioversion (DCCV), repeat ablation, and all-cause hospitalizations. Adverse events were recorded in the registry and included access site complications (i.e., bleeding, groin hematoma, pseudoaneurysm, and arterio-venous fistula), cardiac perforation or tamponade, stroke or transient ischemic attack, pericarditis, myocardial infarction, atrio-esophageal fistula, phrenic nerve paralysis, and pulmonary vein stenosis.

\subsection{Statistical analysis}

The baseline characteristics of those with ER versus no ER were reported as means \pm one standard deviation and frequency $(n$ and $\%)$ for continuous and categorical variables, 
respectively. Chi-square and $t$-tests were used for the betweengroup comparisons of the categorical and continuous variables, respectively. Participant characteristics that differed between those with and without ER in univariate analysis (at a $p$ level $<0.20$ ) were entered into a Cox regression model with backward selection method to identify clinical predictors of ER. A significance level of $p \leq 0.05$ was used for variable removal during the selection process.

Time to recurrence and event-free survival curves were analyzed using the Kaplan-Meier method with a 3-month BP and log-rank significance testing. Cox proportional hazards modeling was used to analyze arrhythmia-free survival with a 3-month BP with results presented as hazard ratios (HR) with $95 \%$ confidence intervals (CI), after verifying proportionality assumptions. Patients who were lost to follow-up were censored at the date of last known follow-up. Variables in the multivariable model were chosen a priori based on the potential for confounding from prior literature and availability within the dataset, including age, sex, body mass index, hypertension, diabetes, obstructive sleep apnea, chronic kidney disease, heart failure, type of atrial fibrillation (paroxysmal vs persistent), roof line ablation, mitral isthmus line ablation, and cavotricuspid isthmus ablation. Finally, receiver operator characteristic (ROC) curve analysis was performed to assess the correlation between the timing of ER and LR. Missing values were minimal (except in the case of the echocardiographic parameters) and roughly equivalent between groups for all variables and were thus omitted. All analyses were performed using IBM SPSS Statistics Version 26 (IBM Corp, NY, USA).

\section{Results}

\subsection{Differences in clinical characteristics in patients with and without early recurrence of atrial tachyarrhythmias}

The baseline characteristics of 259 patients (mean age 63.6 years, $75.3 \%$ male) undergoing repeat PVI are presented in Table 1 . A total of $180(69.5 \%)$ patients had no ER, while $79(30.5 \%)$ patients had ER. There were no significant differences in age, total duration of AF, paroxysmal AF, co-morbidities, or echocardiographic parameters. Patients with ER were more likely to be prescribed an antiarrhythmic drug (AAD) in the BP (initiated or continued at the time of index ablation hospitalization) than those without ER ( $89.9 \%$ vs $75.4 \%$, respectively, $p=0.008$ ). No significant differences were observed between type of AAD prescription or other cardiac medications.

\subsection{Procedural characteristics and complications}

As shown in Table 2, procedure times were significantly longer in those with ER compared to those with no ER (258 \pm 69 vs $218 \pm 66$ mins, respectively, $p<0.001$ ), while no differences were observed with total fluoroscopy time. Pulmonary vein reconnection was present in $75 \%$ of those with and $78 \%$ of those without ER $(p=0.8)$. Those with ER were more likely to have undergone left atrial roof line ablation (46.8\% vs $31.1 \%, p=0.014)$ and mitral isthmus line ablation $(43.0 \%$ vs $25.6 \%, p=0.005)$ than those with no ER. Complication rates were low with no significant differences between those with and without ER.

\subsection{Predictors of ER and LR}

In multivariable Cox regression, there were no significant predictors of ER. Significant predictors of LR after multivariable adjustment included roof line ablation (HR: $1.83,95 \% \mathrm{CI}$ $1.20-2.7, p=0.005$ ) and ER (HR: $2.3,95 \%$ CI 1.62-3.36, $p$ $<0.001)$.

\subsection{Outcomes}

During a median follow-up of 1221 (interquartile range [IQR]: 523-1712) days, LR on or off AAD occurred in 135/259 $(52.1 \%)$. LR occurred in $60 / 79(75.9 \%)$ of those with ER and 75/180 (41.7\%) of those without ER, while 19/124 (15.3\%) of those with ER did not experience LR. The median time to LR was 251 (IQR: 145-1014) days in those with ER compared to 1286 (IQR: 293-2468) days in those without ER. As shown in Fig. 1a, Kaplan-Meier analysis showed a higher risk of LR in those with ER with approximately $78 \%$ of those with ER and $44 \%$ of those without ER experiencing LR at 4 years (log rank $p<0.001)$. After multivariate adjustment, the risk of LR was over twofold higher in those with ER compared to those without ER (HR: 2.36, CI 1.54-3.32, $p<$ 0.001). Furthermore, 168/259 (64.9\%) patients underwent AAD discontinuation after the repeat ablation, including 57/ $79(72.2 \%)$ of those with ER and 111/180 (61.7\%) of those without ER. At 4 years of follow-up, $79 \%$ of those with ER and $46 \%$ of those without ER experienced LR at 4 years (long rank $p=0.003$ ), as shown in Fig. 1b. A strong association was also observed with an increased risk of LR off AAD in those with ER (Table 3).

A total of 55/259 (21.2\%) patients underwent DCCV, including 23/79 (34.2\%) of those with and 28/180 (15.5\%) of those without ER. The mean time to DCCV of those with ER was $1560( \pm 821)$ days and those without ER was 2077 ( \pm 1510 ) days. As shown in Fig. 2a, approximately $25 \%$ of those with ER underwent cardioversion, while $17 \%$ of those without ER underwent DCCV at 4 years (log-rank $p>0.001)$. After multivariate adjustment, the risk of DCCV post-BP was higher in those with ER compared to those without ER (HR: 2.33, CI 1.19-4.56, $p=0.014$; Table 3).

A total of 49/259 (19.1\%) patients underwent repeat ablation, including 29/79 (36.7\%) of those with ER and 20/180 
Table 1 Baseline characteristics of patients with and without recurrence of early atrial tachyarrhythmias (ER) during the 3-month blanking period

\begin{tabular}{|c|c|c|c|}
\hline Variable & $\begin{array}{l}\mathrm{ER} \\
(N=79)\end{array}$ & $\begin{array}{l}\text { No ER } \\
(N=180)\end{array}$ & $p$ value \\
\hline Age, y & $64.3( \pm 9.6)$ & $63.2( \pm 10.7)$ & 0.47 \\
\hline Male & $64(81)$ & $131(72.8)$ & 0.16 \\
\hline Body mass index (kg/min) & $29.4( \pm 5.6)$ & $28.0( \pm 5.4)$ & 0.056 \\
\hline Total duration of AF, days & $2330.4( \pm 1839.3)$ & $2284.7( \pm 1818.5)$ & 0.86 \\
\hline Paroxysmal AF & $49(62.0)$ & $133(73.9)$ & 0.054 \\
\hline AT/AFL & $6(7.6)$ & $15(8.3)$ & 0.87 \\
\hline $\mathrm{CHA}_{2} \mathrm{DSVA}_{2} \mathrm{SC}$ score & $2.1( \pm 1.5)$ & $1.9( \pm 1.5)$ & 0.32 \\
\hline Permanent pacemaker & $6(7.6)$ & $19(10.6)$ & 0.46 \\
\hline Cardiac resynchronization therapy & $3(3.8)$ & $8(4.4)$ & 0.81 \\
\hline \multicolumn{4}{|l|}{ Comorbidities } \\
\hline Hypertension & $44(55.7)$ & $96(3.6)$ & 0.76 \\
\hline Hyperlipidemia & $32(40.5)$ & $63(35.2)$ & 0.42 \\
\hline Diabetes & $8(10.1)$ & $17(9.4)$ & 0.86 \\
\hline Heart failure & $14(17.7)$ & $21(11.7)$ & 0.19 \\
\hline Chronic kidney disease & $1(1.3)$ & $3(1.7)$ & 0.80 \\
\hline Chronic obstructive lung disease & $0(0)$ & $6(4.4)$ & 0.10 \\
\hline Obstructive sleep apnea & $9(11.4)$ & $25(13.9)$ & 0.58 \\
\hline Prior stroke & $11(13.9)$ & $15(8.3)$ & 0.17 \\
\hline Coronary artery disease & $13(16.5)$ & $34(18.9)$ & 0.64 \\
\hline Prior percutaneous coronary intervention & $7(8.9)$ & $20(11.0)$ & 0.59 \\
\hline Prior coronary artery bypass graft surgery & $1(1.3)$ & $6(3.3)$ & 0.35 \\
\hline Smoker & $13(16.5)$ & $27(15.0)$ & 0.77 \\
\hline \multicolumn{4}{|l|}{ Medications } \\
\hline Antiarrhythmic drug during blanking period & $71(89.9)$ & $135(75.4)$ & 0.008 \\
\hline Flecainide & $0(0)$ & $1(0.6)$ & 0.10 \\
\hline Propafenone & $5(6.3)$ & $10(5.6)$ & 0.81 \\
\hline Sotalol & $20(25.3)$ & $47(26.1)$ & 0.89 \\
\hline Dronedarone & $9(11.4)$ & $11(6.1)$ & 0.14 \\
\hline Amiodarone & $10(12.7)$ & $17(9.4)$ & 0.44 \\
\hline Dofetilide & $4(5.1)$ & $8(4.4)$ & 0.83 \\
\hline Beta blocker & $39(49.4)$ & $81(45.0)$ & 0.52 \\
\hline Calcium channel blocker & $18(22.8)$ & $54(30.0)$ & 0.23 \\
\hline Digoxin & $9(11.4)$ & $12(6.7)$ & 0.20 \\
\hline ACEi/ARB & $25(31.6)$ & $35.5(35.6)$ & 0.80 \\
\hline Echocardiographic parameters & $N=32$ & $N=75$ & \\
\hline Left atrial diameter, mm & $4.1( \pm 0.8)$ & $4.3( \pm 0.6)$ & 0.32 \\
\hline Left atrial volume index & $32.8( \pm 15.2)$ & $33.0(7.6)$ & 0.95 \\
\hline Left ventricular ejection fraction & $59.1( \pm 10.4)$ & $61.5( \pm 8.0)$ & 0.38 \\
\hline Left ventricular end diastolic diameter & $4.7( \pm 0.6)$ & $4.9( \pm 0.7)$ & 0.57 \\
\hline
\end{tabular}

Abbreviations: $E R$, early recurrence of atrial tachyarrhythmia; $A F$, atrial fibrillation; $A T$, atrial tachycardia; $A F L$, atrial flutter; $A C E i, \mathrm{ACE}$ inhibitor; $A R B$, angiotensin receptor blocker
$(11.2 \%)$ of those without ER. The mean time to repeat ablation was 1549 days $( \pm 790)$ in those with ER and $2196( \pm$ 1406) days in those without ER. No patients underwent repeat ablation during the BP. Approximately $56 \%$ of those with ER and $21 \%$ of those without ER underwent repeat ablation at 4 years $(\log$-rank $p<0.001)$, as shown in Fig. $2 b$. The risk of repeat ablation was fourfold higher in those with ER compared to those without ER after adjusted analysis (HR: 4.2, CI 2.19-7.81, $p<0.001$; Table 3).

All-cause hospitalizations occurred in 79/259 (30.5\%), including 18/79 (22.8\%) of those with ER and 48/190 (26.7\%) of those without ER. The mean time to hospitalization was 
Table 2 Procedural characteristics and complications in patients with and without subsequent recurrence of early atrial tachyarrhythmia (ER)

\begin{tabular}{llll}
\hline Variable & $\begin{array}{l}\text { ER } \\
(n=79)\end{array}$ & $\begin{array}{l}\text { No ER } \\
(n=180)\end{array}$ & $p$ value \\
\hline Procedural characteristics & & & \\
Total hospital stay (days) & $1.4( \pm 1.0)$ & $1.2( \pm 0.6)$ & 0.10 \\
Total procedure time (mins) & $258( \pm 69)$ & $218( \pm 66)$ & $<0.001$ \\
Fluoroscopy time (mins) & $74.8( \pm 27.2)$ & $64.6( \pm 22.8)$ & 0.28 \\
Pulmonary vein reconnection & $59(74.6)$ & $140(77.8)$ & 0.81 \\
Successful PVI & $76(96.2)$ & $175(97.2)$ & 0.86 \\
Ablation type & & & \\
Segmental & $33(44)$ & $110(63.6)$ & 0.004 \\
Circumferential & $19(25.3)$ & $21(12.1)$ & 0.02 \\
Both & $23(30.7)$ & $42(24.3)$ & 0.35 \\
CTI flutter line & $35(44.3)$ & $78(43.3)$ & 0.83 \\
LA roof line & $37(46.8)$ & $56(31.1)$ & 0.014 \\
Mitral isthmus line & $34(43.0)$ & $46(25.6)$ & 0.005 \\
CFAE ablation & $7(9.1)$ & $10(5.7)$ & 0.33 \\
Procedural complications & & & \\
Access site complication & $9(11.4)$ & $10(5.6)$ & 0.12 \\
Cardiac perforation/tamponade & $0(0)$ & $0(0)$ & NA \\
Stroke/transient ischemic attack & $0(0)$ & $2(1.1)$ & 0.89 \\
Pericarditis & $1(1.3)$ & $1(0.6)$ & 0.36 \\
Other complications* & $0(0)$ & $0(0)$ & NA \\
\hline
\end{tabular}

*Other complications included myocardial infarction, atrioesophageal fistula, phrenic nerve paralysis, and pulmonary vein stenosis

Abbreviations: $P V I$, pulmonary vein isolation; $C T I$, cavotricuspid isthmus; $L A$, left atrial; $C F A E$, complex fractionated atrial electrogram

1775 ( \pm 879$)$ days in those with ER and $1826( \pm 1086)$ days in those without ER with approximately $38 \%$ hospitalization rate at 4 years (log-rank $p=0.38$ ), as shown in Fig. 2c. After multivariable adjustment, there was no significant difference in hospitalizations between those with ER and without ER (HR: 0.94, CI 0.55-1.76, $p=0.94$; Table 3).

Table 3 Cox-proportional hazards models for the association of early recurrence of atrial tachyarrhythmias and long-term outcomes

\begin{tabular}{lllll}
\hline Outcome & $\begin{array}{l}\text { Unadjusted HR } \\
(95 \% \text { CI })\end{array}$ & $p$ value & $\begin{array}{l}\text { Adjusted HR } \\
(95 \% \text { CI })\end{array}$ & $p$ value \\
\hline LR on/off AAD & $2.29(1.63-3.22)$ & $<0.001$ & $2.36(1.54-3.32)$ & $<0.001$ \\
LR off AAD & $2.17(1.41-3.33)$ & $<0.001$ & $2.61(1.56-4.36)$ & $<0.001$ \\
DCCV & $2.60(1.32-5.12)$ & 0.006 & $3.63(1.56-8.46)$ & 0.003 \\
Repeat ablation & $3.63(2.05-6.42)$ & $<0.001$ & $4.20(2.19-7.81)$ & $<0.001$ \\
Hospitalization & $1.22(0.70-2.10)$ & 0.49 & $0.88(0.44-1.77)$ & 0.73 \\
\hline
\end{tabular}

Abbreviations: $C I$, confidence interval; $L R$, late recurrence; $A A D$, antiarrhythmic drug; $D C C V$, direct current cardioversion

\subsection{Significance of ER timing within the blanking period}

Of the 79 patients with ER, the initial timing of ER occurred in the first month in 49 patients, second month in 21 patients, and third month in 9 patients. After adjustment, the risk of LR was highest when ER occurred during the first month (HR: 2.32, CI 1.57-3.74, $p<0.001$ ). A lower risk of LR was observed when ER occurred during the second month (HR: 2.01, CI $1.13-3.83, p=0.019$ ), while no signification association with LR with ER in the third month (HR: 1.46, CI 0.63-3.36, $p=$ 0.374 ), as shown in Table 4. Despite an increased risk with LR when ER occurred earlier in the BP, ROC analysis assessing the relationship between the timing of ER and LR revealed a poor prediction (area under the curve, $0.638, p<0.001$ ). The point on the ROC curve with best discriminatory potential was 1 day (sensitivity $43.3 \%$, specificity $83.1 \%$ ).

\section{Discussion}

We demonstrate several key findings in the first study examining the relationship between ER and long-term outcomes in patients following repeat ablation. First, over a median followup period of 3.2 years, approximately one-third of patients will experience ER following repeat ablation and the vast majority $(76 \%)$ of those with ER will have LR after repeat ablation. Furthermore, less than a quarter of those with ER were free from LR at 4 years, compared to $56 \%$ of those without ER. Second, there were no predictors of ER, while ER was the strongest predictor of LR. Third, ER was strongly associated with LR, cardioversion, and repeat ablation, but not hospitalization after multivariable adjustment. Lastly, although the majority of ER occurred within the first month of the $\mathrm{BP}$ and was associated with over a twofold increased risk of LR, the prediction of LR based on ER timing within the BP was poor. Taken together, these findings highlight the increased risk of long-term ablation failure in those with ER and challenges clinical utilization of the 3-month BP in patients following repeat ablation.

Previous studies have demonstrated an ER incidence of approximately $38 \%$ within the 3-month BP in patients after de novo ablation [2]. Although approximately one-half of patients with ER may not experience LR, overwhelming data suggests that the risk of LR remains significantly higher in those with ER $[2,5,6]$. Among de novo ablations, the relationship persists across varying ablation strategies, including radiofrequency ablation and cryoablation, and in those with paroxysmal and persistent AF $[4,8,11]$. To the best of our knowledge, the present study is the first to examine the association between ER and LR after repeat ablation. We observed a similar incidence of ER after repeat ablation as compared to reports following de novo ablation; however, only less than a 


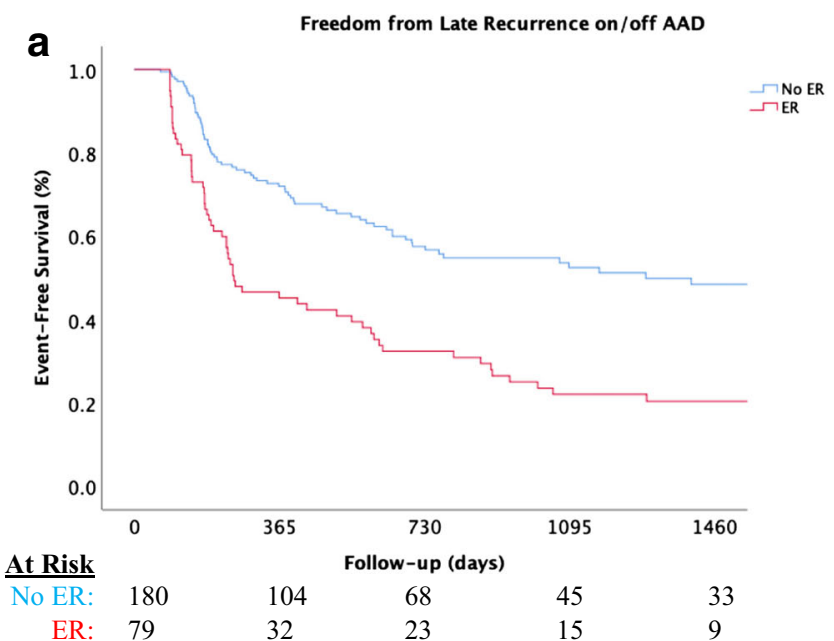

Fig. 1 Title: Kaplan-Meier plots comparing clinical outcomes stratified by early recurrence of atrial tachyarrhythmias within the blanking period versus no early recurrence of atrial tachyarrhythmias during the blanking b

Freedom from LR off AAD

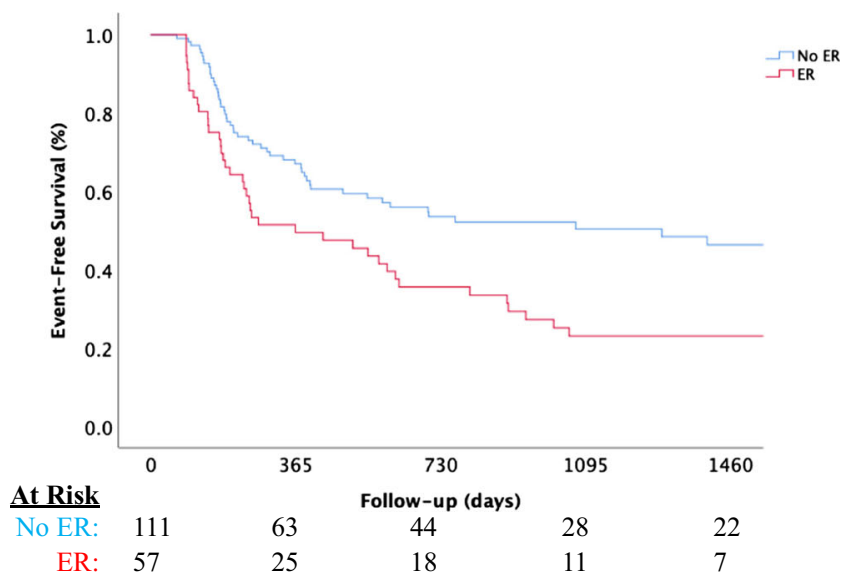

period. a Freedom from late recurrence (LR) on or off anti-arrhythmic drug (AAD); b Freedom from LR off AAD. Caption: Time zero indicates $91^{\text {st }}$ day (after blanking period)
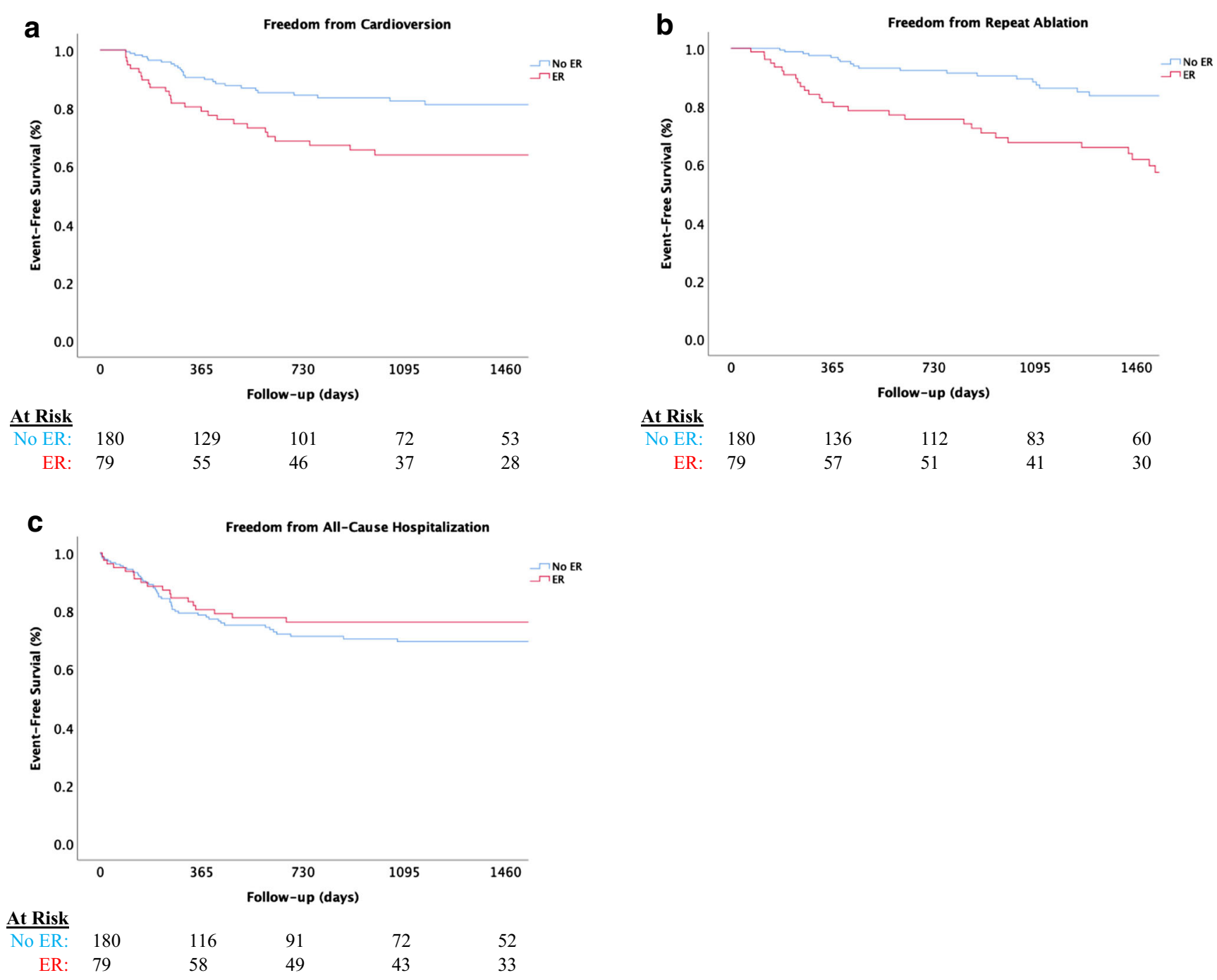

Fig. 2 c Freedom from cardioversion; $\mathbf{d}$ Freedom from repeat ablation; e Freedom from all-cause hospitalization. Caption: Time zero indicates day of procedure. 
Table 4 Association of early recurrence of ER and LR according to timing within the blanking period

\begin{tabular}{lllllll}
\hline Group & $N$ & LR $\%$ & $\begin{array}{l}\text { Unadjusted HR } \\
(95 \% \text { CI })\end{array}$ & $p$ value & Adjusted HR (95\% CI) & $p$ value \\
\hline No ER & 177 & 41.8 & Reference & -- & Reference & -- \\
Month 1 & 48 & 79.2 & $2.31(1.56-3.43)$ & $<0.001$ & $2.32(1.57-3.74)$ & $<0.001$ \\
Month 2 & 23 & 65.2 & $2.21(1.27-3.85)$ & 0.005 & $2.01(1.13-3.83)$ & 0.02 \\
Month 3 & 11 & 77.8 & $1.76(0.81-2.83)$ & 0.15 & $1.46(0.63-3.36)$ & 0.37 \\
\hline
\end{tabular}

Abbreviations: $C I$, confidence interval; $E R$, early recurrence of atrial tachyarrhythmia; $L R$, late recurrence quarter of those with ER will not experience LR in our cohort, negating the notion that ER is benign following repeat ablation; in fact, our findings may suggest that any recurrence after repeat ablation may have clinical significance.

It has been shown that repeat ablation improves freedom from LR with approximately three-quarters of patients free of LR at 1 year with a steady decline thereafter $[12,13]$. In our cohort, ER after repeat ablation was associated with over a twofold increase in LR (both on/off ADD and off AAD) over the study period. At 1 year, approximately three-quarters of those without ER were free of LR, compared to only $45 \%$ of those with ER. Various predictors of LR following repeat ablation have been previously described, such as persistent $\mathrm{AF}$, hypertension, enlarged left atrium, valvular disease, and heart failure; however, the relationship with ER has not been examined $[14,15]$. We found ER to be the strongest predictor of LR, along with roof line ablation. Whether the additional lesions contribute to LR or are a marker for a more advanced arrhythmogenic substrate is unclear.

Since the 3-month BP was created arbitrarily, efforts to elucidate the significance of ER within the period after de novo ablation have generally demonstrated that later recurrence within the $\mathrm{BP}$ portends to worse prognosis [5]. Willems et al. stratified 401 patients according to the last episode of ER within the BP. Those with last recurrence within the third month were associated with HR of 9.4 in predicting LR compared to a HR of 1.84 in the first month. Further ROC analysis identified 50 days as the optimal BP duration [8]. Similarly, Olshausen et al. identified 46 days as the optimal BP duration based on last episode of ER in a cohort of 713 patients who had undergone de novo PVI. In a Danish registry of 7339 patients, Hodges et al. demonstrated a significant graded increase of odds of LR according to the first occurrence of ER stratified by month (OR 2.08, 4.96, 5.14) [16]. While we also assessed ER according to first occurrence, we found divergent results. The majority of the patients in our cohort with ER (60\%) experienced ER within the first month and were at highest risk for LR as compared to the second and third months. However, the correlation between timing of ER and LR was poor based on the ROC analysis, likely influenced by the small sample size and few event rates in the second and third months. Still, these findings suggest that patients with ER after repeat ablation typically experience ER immediately after the procedure and may be considered a failed ablation even during the BP. This may question the clinical utility of the traditional 3-month BP in patients undergoing repeat ablation.

The mechanism underlying ER following repeat ablation remains unknown. Prior studies following patients after de novo ablation have suggested that inflammation post-ablation, incomplete pulmonary vein isolation, non-PV triggers, or inappropriate time allotment for completion of electroanatomic changes may contribute to $\operatorname{ER}[3,7,17,18]$. Given the strong association between ER and LR, particularly during the first month, it argues that transient effects may have little influence on the risk relationship. In our cohort, the pulmonary veins reconnected in only approximately a quarter of patients at the time of the repeat ablation. These findings suggest that pulmonary vein isolation alone may not be sufficient to control AF in select patients as the arrhythmogenic substrate advances. Furthermore, we have shown that ER also increases the risk of cardioversion and another repeat ablation after the BP. Although studies after first-time ablation have shown that those undergoing early re-ablation strategy have improved LR freedom, further invasive rhythm control strategy for AF in patients who have already undergone repeat ablation may not always be the appropriate treatment [4, 19]. Modifiable factors such as weight loss, increasing physical activity, and reducing alcohol intake should be revisited and encouraged [20]. Additionally, further research aimed at identifying, advancing, and treating factors influencing failure of ablation, such as recognizing irreversible myopathic changes, triggers not amenable to current ablation techniques or observed with current mapping devices, or discovering unknown underlying AF mechanisms is warranted.

Our study must be interpreted in the context of several limitations inherent to its design. First, as a retrospective observational study, causal inferences cannot be made. Yet, these findings highlight a high-risk group that could benefit from further investigation. Second, the multivariable models were adjusted for available risk factors and characteristics. Other factors that may play a role in predicting outcomes, such as echocardiography characteristics, contact force sensing catheter use, and type of ER (AF vs AFL vs AT) were 
excluded due to missing values. Third, while all patients underwent PVI during the first ablation, data regarding adjunctive lesions from the first procedure were not captured. Finally, the type of post-ablation arrhythmia monitoring likely varied in the cohort as it was not standardized. Although this may underestimate the incidence of ER and LR, we believe that the findings remain valid as it reflects clinical practice.

\section{Conclusion}

Following repeat $\mathrm{AF}$ ablation, ER is frequently observed and strongly associated with LR, cardioversion, repeat ablation, but not hospitalization. The majority of ER occurred within the first month of the BP and was associated with the highest risk of LR, although the prediction of ER timing within the BP was poor, questioning the applicability of the standard 3month BP to AF patients following repeat ablation. Further studies are warranted to identify potential treatment strategies to reduce LR in this high-risk cohort.

\begin{abstract}
Abbreviations AF, Atrial fibrillation; ER, Early recurrence of atrial tachyarrhythmia; PVI, Pulmonary vein isolation; LR, Late recurrence of atrial tachyarrhythmia; UCSD, University of California, San Diego; AF, Atrial flutter; AT, Atrial tachycardia; DCCV, Direct current cardioversion; ROC, Receiver operator characteristic; AAD, Antiarrhythmic drug
\end{abstract}

\section{Declarations}

Ethics approval The study was approved by the Institutional Review Board of University of California, San Diego.

Conflict of interest Dr. Hsu reports receiving honoraria from Medtronic, Abbott, Boston Scientific, Biotronik, Janssen Pharmaceuticals, BristolMyers Squibb, Altathera Pharmaceuticals, Zoll Medical, and BiosenseWebster; equity in Acutus Medical and Vektor Medical; and research grants from Biotronik and Biosense-Webster. Dr. Ho receives grant support from the American Heart Association (AHA 19CDA34760021), National Institutes of Health (NIH 1KL2TR001444), and reports equity in Vektor Medical Inc. unrelated to this work. Dr. Krummen reports receiving a research grant from the University Galvanizing Engineering in Medicine Foundation, equity in Vektor Medical unrelated to the present work, and fellowship support from Abbott, Biosense-Webster, Biotronik, Boston Scientific, and Medtronic. Dr. Feld reports equity in Acutus Medical, Inc., Vektor Medical, Inc., and Perminova, Inc.; has received honoraria from Altathera Pharmaceuticals, Inc., Vektor Medical, Inc., and Acutus Medical, Inc.; and, as EP Fellowship Training Program Director, indirectly receives fellowship stipend support from Abbott, Inc., Boston Scientific, Inc., Biotronik, Inc., and Biosense Webster, Inc.

Open Access This article is licensed under a Creative Commons Attribution 4.0 International License, which permits use, sharing, adaptation, distribution and reproduction in any medium or format, as long as you give appropriate credit to the original author(s) and the source, provide a link to the Creative Commons licence, and indicate if changes were made. The images or other third party material in this article are included in the article's Creative Commons licence, unless indicated otherwise in a credit line to the material. If material is not included in the article's Creative Commons licence and your intended use is not permitted by statutory regulation or exceeds the permitted use, you will need to obtain permission directly from the copyright holder. To view a copy of this licence, visit http://creativecommons.org/licenses/by/4.0/.

\section{References}

1. Calkins H, Hindricks G, Cappato R, Kim YH, Saad EB, Aguinaga L, et al. 2017 HRS/EHRA/ECAS/APHRS/SOLAECE expert consensus statement on catheter and surgical ablation of atrial fibrillation: executive summary. Heart Rhythm. 2017;14(10):e445-e94. https://doi.org/10.1016/j.hrthm.2017.07.009.

2. Andrade JG, Khairy P, Verma A, Guerra PG, Dubuc M, Rivard L, et al. Early recurrence of atrial tachyarrhythmias following radiofrequency catheter ablation of atrial fibrillation. Pacing Clin Electrophysiol. 2012;35(1):106-16. https://doi.org/10.1111/j. 1540-8159.2011.03256.x.

3. Lim HS, Schultz C, Dang J, Alasady M, Lau DH, Brooks AG, et al. Time course of inflammation, myocardial injury, and prothrombotic response after radiofrequency catheter ablation for atrial fibrillation. Circ Arrhythm Electrophysiol. 2014;7(1):83-9. https://doi.org/10.1161/CIRCEP.113.000876.

4. Andrade JG, Khairy P, Macle L, Packer DL, Lehmann JW, Holcomb RG, et al. Incidence and significance of early recurrences of atrial fibrillation after cryoballoon ablation: insights from the multicenter Sustained Treatment of Paroxysmal Atrial Fibrillation (STOP AF) Trial. Circ Arrhythm Electrophysiol. 2014;7(1):69-75. https://doi.org/10.1161/CIRCEP.113.000586.

5. Themistoclakis S, Schweikert RA, Saliba WI, Bonso A, Rossillo A, Bader $\mathrm{G}$, et al. Clinical predictors and relationship between early and late atrial tachyarrhythmias after pulmonary vein antrum isolation. Heart Rhythm. 2008;5(5):679-85. https://doi.org/10.1016/j. hrthm.2008.01.031.

6. Choi JI, Pak HN, Park JS, Kwak JJ, Nagamoto Y, Lim HE, et al. Clinical significance of early recurrences of atrial tachycardia after atrial fibrillation ablation. J Cardiovasc Electrophysiol. 2010;21(12):1331-7. https://doi.org/10.1111/j.1540-8167.2010. 01831.x.

7. Das M, Wynn GJ, Morgan M, Lodge B, Waktare JE, Todd DM, et al. Recurrence of atrial tachyarrhythmia during the second month of the blanking period is associated with more extensive pulmonary vein reconnection at repeat electrophysiology study. Circ Arrhythm Electrophysiol. 2015;8(4):846-52. https://doi.org/10.1161/ CIRCEP.115.003095.

8. Willems S, Khairy P, Andrade JG, Hoffmann BA, Levesque S, Verma A, et al. Redefining the blanking period after catheter ablation for paroxysmal atrial fibrillation: insights from the ADVICE (Adenosine Following Pulmonary Vein Isolation to Target Dormant Conduction Elimination) Trial. Circ Arrhythm Electrophysiol. 2016;9(8). https://doi.org/10.1161/CIRCEP.115. 003909.

9. Liang JJ, Elafros MA, Chik WW, Santangeli P, Zado ES, Frankel DS, et al. Early recurrence of atrial arrhythmias following pulmonary vein antral isolation: timing and frequency of early recurrences predicts long-term ablation success. Heart Rhythm. 2015;12(12): 2461-8. https://doi.org/10.1016/j.hrthm.2015.07.015.

10. Tilz RR, Rillig A, Thum AM, Arya A, Wohlmuth P, Metzner A, et al. Catheter ablation of long-standing persistent atrial fibrillation: 5 -year outcomes of the Hamburg Sequential Ablation Strategy. J Am Coll Cardiol. 2012;60(19):1921-9. https://doi.org/10.1016/j. jacc.2012.04.060. 
11. Andrade JG, Macle L, Khairy P, Khaykin Y, Mantovan R, De Martino G, et al. Incidence and significance of early recurrences associated with different ablation strategies for AF: a STAR-AF substudy. J Cardiovasc Electrophysiol. 2012;23(12):1295-301. https://doi.org/10.1111/j.1540-8167.2012.02399.x.

12. Bhargava M, Di Biase L, Mohanty P, Prasad S, Martin DO, Williams-Andrews M, et al. Impact of type of atrial fibrillation and repeat catheter ablation on long-term freedom from atrial fibrillation: results from a multicenter study. Heart Rhythm. 2009;6(10): 1403-12. https://doi.org/10.1016/j.hrthm.2009.06.014.

13. Ouyang F, Tilz R, Chun J, Schmidt B, Wissner E, Zerm T, et al. Long-term results of catheter ablation in paroxysmal atrial fibrillation: lessons from a 5-year follow-up. Circulation. 2010;122(23): 2368-77. https://doi.org/10.1161/CIRCULATIONAHA.110. 946806.

14. Weerasooriya R, Khairy P, Litalien J, Macle L, Hocini M, Sacher F, et al. Catheter ablation for atrial fibrillation: are results maintained at 5 years of follow-up? J Am Coll Cardiol. 2011;57(2):160-6. https:// doi.org/10.1016/j.jacc.2010.05.061.

15. Rostock T, Salukhe TV, Steven D, Drewitz I, Hoffmann BA, Bock $\mathrm{K}$, et al. Long-term single- and multiple-procedure outcome and predictors of success after catheter ablation for persistent atrial fibrillation. Heart Rhythm. 2011;8(9):1391-7. https://doi.org/10. 1016/j.hrthm.2011.04.012.

16. Hodges G, Bang CN, Torp-Pedersen C, Hansen ML, Schjerning AM, Hansen J, et al. Significance of early recurrence of atrial fibrillation after catheter ablation: a nationwide Danish cohort study. J Interv Card Electrophysiol. 2020;60:271-8. https://doi. org/10.1007/s10840-020-00741-x.

17. Liang JJ, Dixit S. Early recurrences during the blanking period after atrial fibrillation ablation. J Atr Fibrillation. 2018;10(5):1726. https://doi.org/10.4022/jafib.1726.

18. Mujović N, Marinković M, Marković N, Vučićević V, Lip GYH, Bunch TJ, et al. The relationship of early recurrence of atrial fibrillation and the 3-month integrity of the ablation lesion set. Sci Rep. 2018;8(1):9875. https://doi.org/10.1038/s41598-018-28072-y.

19. Lellouche N, Jaïs P, Nault I, Wright M, Bevilacqua M, Knecht S, et al. Early recurrences after atrial fibrillation ablation: prognostic value and effect of early reablation. J Cardiovasc Electrophysiol. 2008;19(6):599-605. https://doi.org/10.1111/j.1540-8167.2008. 01188.x.

20. Aldaas OM, Lupercio F, Han FT, Hoffmayer KS, Krummen D, Ho $\mathrm{G}$, et al. Meta-analysis of effect of modest $(\geq 10 \%)$ weight loss in management of overweight and obese patients with atrial fibrillation. Am J Cardiol. 2019;124(10):1568-74. https://doi.org/10. 1016/j.amjcard.2019.08.009.

Publisher's note Springer Nature remains neutral with regard to jurisdictional claims in published maps and institutional affiliations. 